\title{
Vehicle-to-Vehicle Visible Light Communication: How to select receiver locations for optimal performance?
}

\author{
Hossien B. Eldeeb and Murat Uysal \\ Department of Electrical and Electronics Engineering \\ Özyeğin University, Istanbul, Turkey, 34794
}

\begin{abstract}
Visible light communication (VLC) utilizes vehicle headlights as wireless transmitters and has emerged as a strong candidate for vehicle-to-vehicle (V2V) communications. In majority of works on V2V VLC systems, a common underlying assumption is that two vehicles within the same lane follow each other in a perfect alignment. In practical scenarios, a precise alignment is not available between two vehicles even if they are in the same lane. In this paper, we consider a VLC-based V2V system where the destination vehicle is equipped with four photodetectors for an omni-directional coverage. We use ray tracing simulations for channel modelling and calculate the received power for practical cases with imperfect alignment between two vehicles. In addition, we investigate the performance for lane changes. Our simulation results provide insight into which photodetectors are essential for each scenario under consideration.
\end{abstract}

\section{Introduction}

Visible light communication utilizes light emitting diodes (LEDs) as wireless transmitters without any adverse effect on illumination levels [1]. VLC was originally proposed as an indoor wireless access technology to take advantage of the existing illumination infrastructure. The increasing adoption of LED-based headlamps and taillights as well as the availability of LED-based street and traffic lights makes VLC also a strong candidate for vehicle-to-vehicle $(\mathrm{V} 2 \mathrm{~V})$ and vehicle-toinfrastructure (V2I) communications [2, 3].

As in any other communication system, channel modeling is critical for link budget calculations and optimized system design for vehicular VLC [4]. Initial works in the related literature have made some ideal, yet unrealistic assumptions. For example, a common assumption [5-7] is the Lambertian illumination pattern of light source. Such a pattern might be valid for indoor light sources but cannot be used to model the asymmetrical intensity distribution of vehicle headlights. Another overlooked issue is road reflectance and its effect has been studied only in some recent works $[8,9]$. Since vehicular VLC systems operate in outdoor medium, their performance in adverse weather conditions such as rain and fog are also important. These have been further investigated in $[10,11]$.

In majority of works on V2V VLC systems, a common underlying assumption is that two vehicles within the same lane follow each other in a perfect alignment. It is also commonly assumed that there are one or two photodetectors placed at the back of the preceding car. In practical scenarios, it is obvious that there will not be a precise alignment between two vehicles even if they are in the same lane. Similarly, it is important to maintain connectivity while changing a lane. In this context, a major design challenge is how to select a minimum number of photodetectors (receivers) with proper locations to achieve a reliable communication in such practical scenarios.

In this paper, we attempt to answer this fundamental question through realistic channel modeling of VLC-based V2V link. The source destination uses high-beam headlights as its transmitters. We assume that the destination vehicle is equipped with four PDs. The first two PDs are located at the back of the vehicle under taillights. The other two PDs are located at the vehiclesides below the mirrors, one at the left side and the second at the right one. Specifically, we consider three scenarios: 1) As a benchmark, we consider the commonly assumed scenario where two vehicles within the same lane follow each other at a constant speed with perfect alignment. 2) In the second scenario, two vehicles move in the same lane, but are not strictly aligned. We assume a horizontal shift between two vehicles. 3) In the third scenario, the source vehicle changes its lane while communicating with the destination vehicle in the target lane. We run ray tracing simulations to obtain channel impulse response (CIR) for these scenarios. Based on these CIRs, the total received optical power and the power percentage of each PD are calculated. These provide insight into which PDs are essential for each scenario under consideration.

The rest of the paper is organized as follows. In Section II, we describe our channel modeling approach based on nonsequential ray tracing. In Section III, we present the simulations for V2V scenarios. First, we calculate the received power using each individual PD. Then, we present the total contribution of received optical power considering all PDs under consideration. In last part of this section, we introduce the power percentage of each PD along the whole inter-vehicle distance under consideration. Finally, we provide concluding remarks in Section VI.

\section{Channel Modeling Approach and System Model}

For channel modeling, we use built-in non-sequential ray tracing feature of the optic system design software Zemax ${ }^{\circledR}$. This modeling approach was first used to model indoor VLC channels [12] and also applied to V2V channels in [9, 11]. As illustrated in Fig. 1, we consider three V2V scenarios in a twolane highway road, each has a lane width of $3.75 \mathrm{~m}[13,14]$. In Scenario I, we assume that two vehicles are located at the center 


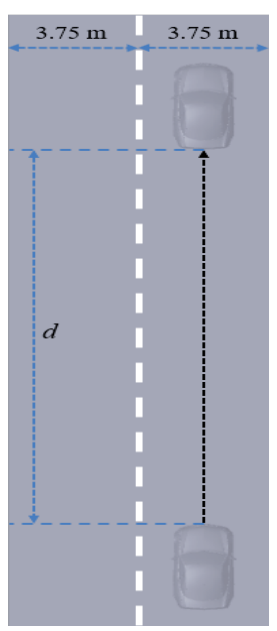

(a)

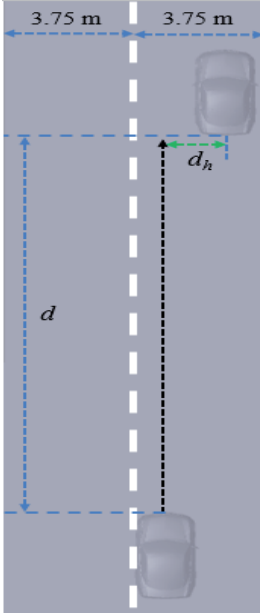

(b)

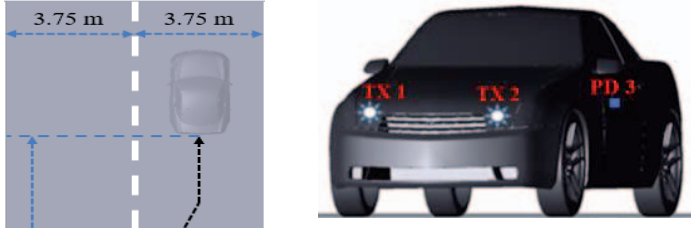

(a)

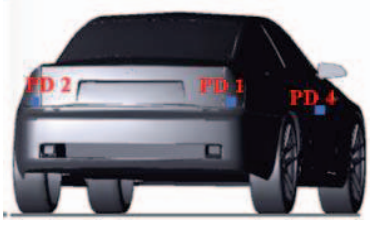

(b)
Fig. 2. Locations of high-beam headlights (transmitters) and photodetectors (receivers) on the vehicles.

TABLE I. Simulation parameters

\begin{tabular}{|c|l|l|}
\hline $\begin{array}{c}\text { Transmitter } \\
\text { specifications }\end{array}$ & $\begin{array}{l}\text { Type: } \\
\text { Brand: } \\
\text { Power }(\mathrm{Pt}):\end{array}$ & $\begin{array}{l}\text { Philips Luxeon white light } \\
\text { high-beam headlamp } \\
1 \mathrm{~W} \text { (normalized) }\end{array}$ \\
\hline Receiver & Area: & $1 \mathrm{~cm} 2$ \\
specifications & FOV: & $180^{\circ}$ \\
\hline \multirow{2}{*}{ Road } & Type: & R2 \\
specifications & Material: & Asphalt \\
& Lane width: & $3.75 \mathrm{~m}$ \\
\hline \multirow{2}{*}{ Car } & Length: & $4.67 \mathrm{~m}$ \\
specifications & Width: & $1.85 \mathrm{~m}$ \\
& Height: & $1.37 \mathrm{~m}$ \\
& Material: & Black gloss paint \\
\hline
\end{tabular}

\section{Simulation of V2V Scenarios}

In this section, we first demonstrate the received optical power with respect to the inter-vehicle distance of $d$ considering the total contribution of all PDs under consideration. Then, we present the power percentage of each PD along the whole intervehicle distance for all scenarios under consideration.

In Fig. 3, we consider scenario II and investigate the effect of misalignment between the vehicles. As a benchmark, perfect alignment scenario (Scenario I) is included. It is observed that the received power reduces when the horizontal displacement increases. For example, consider $d=10 \mathrm{~m}$. The received power when the two vehicles are perfectly aligned $\left(d_{h}=0 \mathrm{~m}\right)$ is -41.4 $\mathrm{dB}$. This reduces to $-42.4 \mathrm{~dB}$ and $-43.6 \mathrm{~dB}$ for $d_{h}=1 \mathrm{~m}$ and $d_{h}=2 \mathrm{~m}$, respectively.

In order to identify which PDs contribute at what extent to total received power, we present pie charts in Fig. 4. It is observed that, in the perfect alignment case, PD1 and PD2 have almost identical shares. Only a very small received power is collected by PD3 and PD4 as a result of reflections. When there is some displacement (to the left-hand side) between two vehicles, it is observed that PD3 (located on left hand side of the destination vehicle) is now able to collect a significant amount of received power. On the other hand, the received power using PD 4 (located on right hand side of destination vehicle) is much reduced due to its location on the other side of displacement.

In Fig. 5, we consider lane changing scenario, i.e. Scenario III. In this scenario, we consider two cases: In the first case (Case A), the source vehicle is located at the center of the lane when it starts to change its lane. In the second case (Case B), the source vehicle is located at the edge of the lane at the start of the lane change process. We assume that the source vehicle starts to change its lane at $d=30 \mathrm{~m}$ and ends this process at $d=15 \mathrm{~m}$. It 


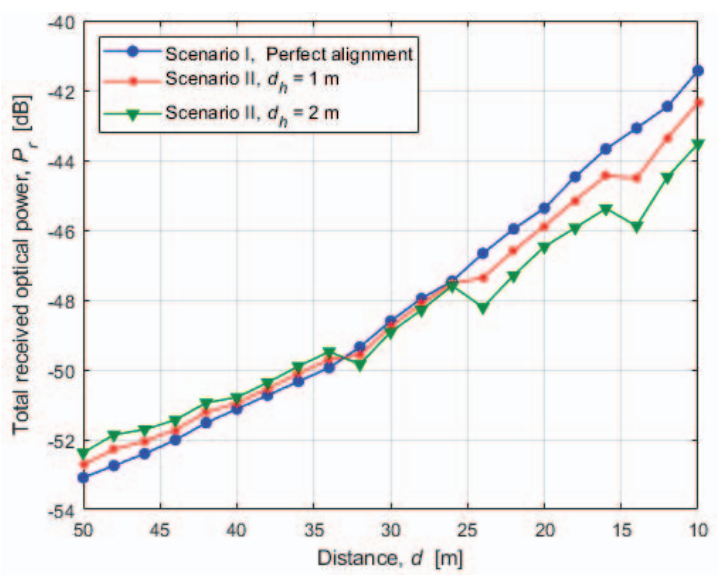

Fig. 3. Received optical power for scenario II (Effect of horizontal displacement).

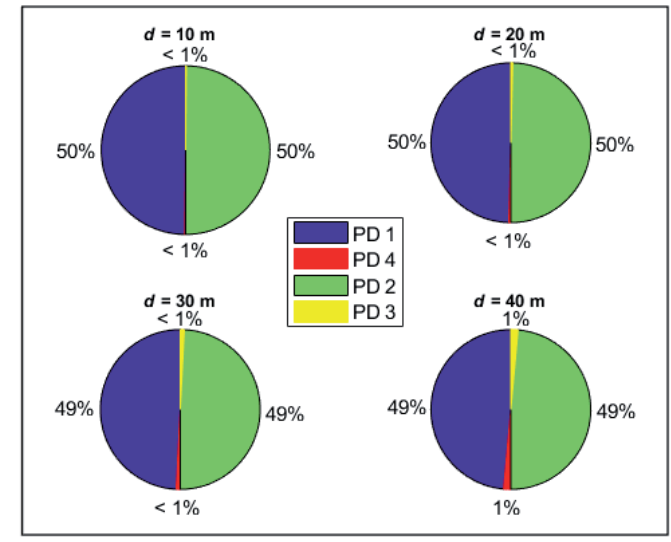

(a) Scenario I

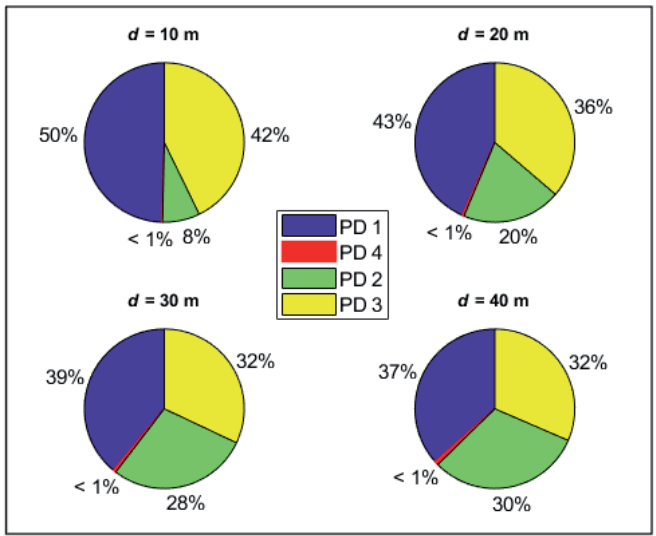

(b) Scenario II

Fig. 4. Power percentage of each PD for Scenarios I and II.

is observed that the rate of change in the received power during the lane changing range (from $15 \mathrm{~m}$ to $30 \mathrm{~m}$ ) is much higher than that in trailing period (from $30 \mathrm{~m}$ to $50 \mathrm{~m}$ ). This is due to that during the lane switching, there is a change in both intervehicle distance $(d)$ and horizontal displacement one $\left(d_{h}\right)$. It can be also observed from the figure that the starting point of the

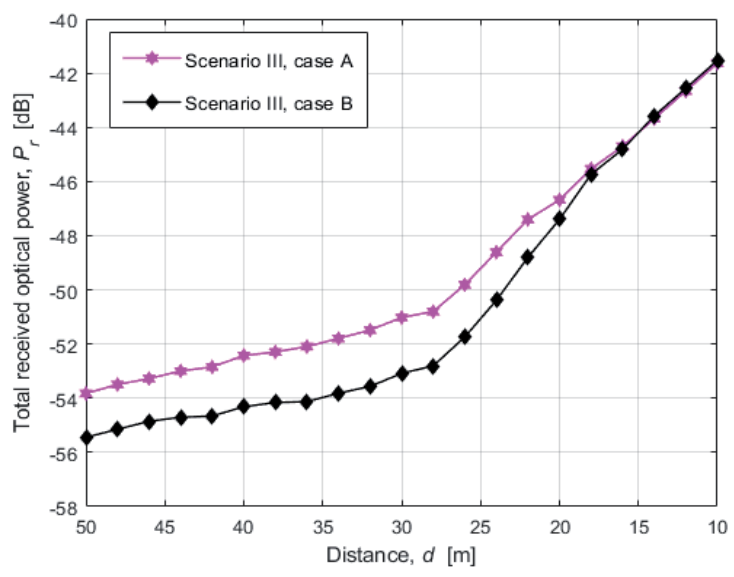

Fig. 5. Received optical power for scenario III (Lane changing).

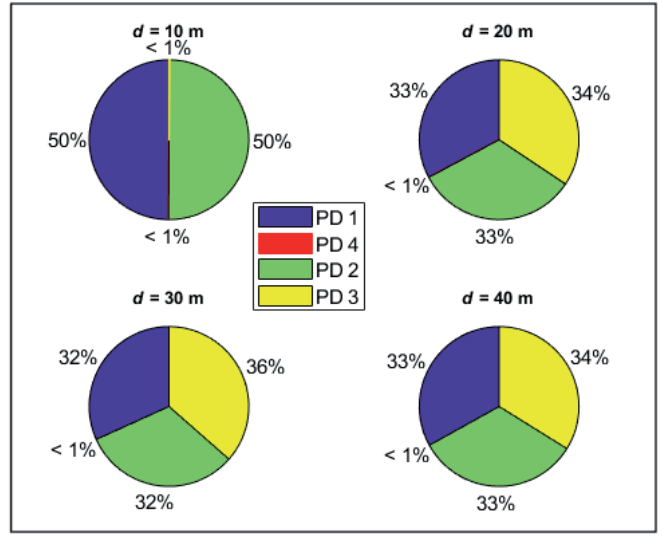

(a) Scenario III, case A

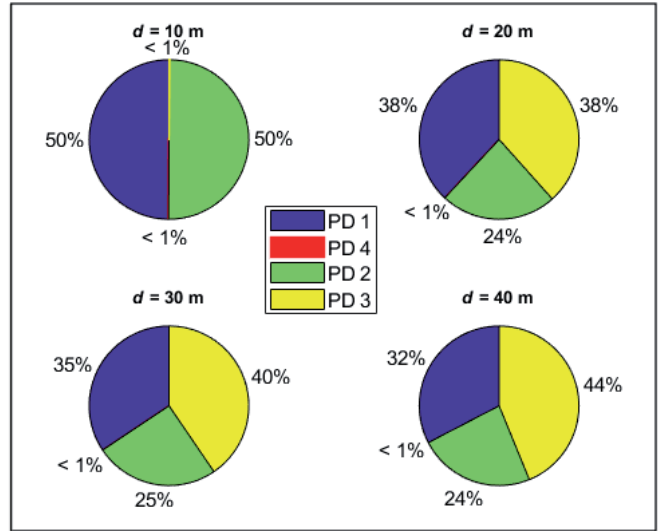

(b) Scenario III, case B

Fig. 6. Power percentage of each PD for all scenarios III.

lane change can also influence on the received power. For example, consider $d=30 \mathrm{~m}$. The received power considering the starting point at the center of the lane (case A) is $-51 \mathrm{~dB}$. For case $\mathrm{B}$ where the starting point at the end/edge of the lane, this value is reduced to $-53.2 \mathrm{~dB}$.

In Fig. 6, we present pie charts to illustrate the percentage of received powers for Scenario III. We assume the lane changing 
occurs from the left-hand side to the right-hand side. It is observed that at the initialization range (from $10 \mathrm{~m}$ to $15 \mathrm{~m}$ ), PD 1 and PD 2 can collect almost all power due to the perfect alignment between the two vehicles. However, during the lane changing range (from $15 \mathrm{~m}$ to $30 \mathrm{~m}$ ), and trailing range (from 30 $\mathrm{m}$ to $50 \mathrm{~m}$ ), PD 3 can collect the highest amount of received power while the contributions of PD 1 and PD 2 are much reduced. It can be also observed in this range (from $15 \mathrm{~m}$ to 50 $\mathrm{m})$ that PD 4 is no longer able to receive power. This is due to its location on the other side of the destination vehicle.

\section{Conclusions}

In this paper, we considered a VLC-based V2V link where the source destination uses high-beam headlights as its transmitters and the destination vehicle is equipped with four PDs. We used non-sequential ray tracing to obtain CIRs and calculate the received power for various practical scenarios including imperfect alignment and lane changes. Our results reveal that two PDs (denoted by PD 1 and PD 2) located at the back of the vehicle under taillights are sufficient to collect almost all power for the perfect alignment case. On the other hand, the other PDs (either PD3 or PD4) become critical when there is imperfect alignment. Our results further reveal that the exact distribution of received power among PDs depend on the inter-distance between vehicles and horizontal displacement. It can be also concluded that four PDs would be sufficient to create the required omni-directional coverage for $\mathrm{V} 2 \mathrm{~V}$ connectivity. It would be a future research direction whether or not there is a need for additional PDs to enable V2I connectivity.

\section{Acknowledgements}

The work of Hossien B. Eldeeb has been supported by H2020 Marie Sklodowska-Curie Innovative Training Network (ITNVisIoN) under Grant 764461. The work of M. Uysal has been supported by the Turkish Scientific and Research Council (TUBITAK) under Grant 215E311.

\section{References}

[1] P. H. Pathak, X. Feng, P. Hu, and P. Mohapatra, "Visible light communication, networking, and sensing: A survey, potential and challenges," IEEE Commun. Surveys Tuts., vol. 17, no. 4, pp. 2047-2077, 2015.

[2] M. Uysal, Z. Ghassemlooy, A. Bekkali, A. Kadri and H. Menouar, "Visible light communication for vehicular networking: Performance study of a V2V system using a measured headlamp beam pattern model," IEEE Veh. Technol. Mag., vol. 10, no. 4, pp. 45-53, Dec. 2015.

[3] A.-M. Cailean and M. Dimian, "Impact of ieee 802.15.7 standard on visible light communications usage in automotive applications," IEEE Commun. Mag., pp. 2-7, 2017.

[4] A. Căilean and M. Dimian, "Current challenges for visible light communications usage in vehicle applications: a survey," IEEE Commun. Surveys Tuts., vol. 19, no. 4, pp. 2681-2703, 2017.

[5] N. Kumar, D. Terra, N. Lourenco, L. N. Alves, and R. L. Aguiar, "Visible light communication for intelligent transportation in road safety applications", Proc. IEEE Int.
Conf. Wireless Commun. Mobile Computing, pp. 1513$1518,2011$.

[6] Y Bao, Y Wang, J Yu, J Shen, "A visible light communication based vehicle collision avoiding system," 15th Int. Conf. Opt. Commun. and Netw. (ICOCN), Hangzhou, pp. 1-3, 2016.

[7] Z. Cui, P. Yue, X. Yi, and J. Li, "Research on nonuniform dynamic vehicle-mounted VLC with receiver spatial and angular diversity," 2019 IEEE Int. Conf. Commun. (ICC), May 2019, pp. 1-7.

[8] B. Turan, G. Gurbilek, A. Uyrus and S. C. Ergen, "Vehicular VLC frequency domain channel sounding and characterization," IEEE Veh. Netw. Conf. (VNC), Taipei, Taiwan, pp. 1-8, 2018.

[9] M. Elamassie, M. Karbalayghareh, F. Miramirkhani, R. C. Kizilirmak, and M. Uysal, "Effect of fog and rain on the performance of vehicular visible light communications", IEEE 87th Veh. Technol. Conf. (VTC2018-Spring), Porto, Portugal, June 2018.

[10] D. Schulz, V. Jungnickel, S. Das, J. Hohmann, J. Hilt, P. Hellwig,A. Paraskevopoulos, and R. Freund, "Long-term outdoor measurementsusing a rate-adaptive hybrid optical wireless/60 ghz link over $100 \mathrm{~m}$," 2017 19th Int. Conf. on Transparent Opt. Netw. (ICTON). IEEE, pp. 1-4, 2017.

[11] H. B. Eldeeb, F. Miramirkhani and M. Uysal, "A Path Loss Model for Vehicle-to-Vehicle Visible Light Communications," 2019 15th Int. Conf. Telecommun. (ConTEL), Graz, Austria, 2019, pp. 1-5.

[12] F. Miramirkhani and M. Uysal, "Channel modeling and characterization for visible light communications," IEEE Photon. J., vol. 7, no. 6, pp. 1-16, 2015.

[13] Schmidt, S. Kharrazi, S. Erlingsson, C. Van Geem, X. Cocu, and B. Jacob, "Falcon ii: Input for a european pbs definition: Review of vehicle legislations and infrastructure design criteria," 15th Interna-tional Symposium on Heavy Vehicule Transport Technology: HVTT 15, Oct 2018, ROTTERDAM, France, 2018.

[14] Vadeby, G. Sorensen, A. Bolling, X. Cocu, P. Saleh, M. Aleksa,F. La Torre, A. Nocentini, and P. Tucka, "Towards a european guide-line for speed management measures in work zones, "Transportation research procedia, vol. 14, pp. 3426-3435, 2016. 